Proceedings

\title{
Physical-Chemical Properties of the Phenolic Compounds of Humulus lupulus and Aromatic Plant Terpenes: Potential for Use in a Cosmetic Formulation ${ }^{\dagger}$
}

\author{
Briolanja dos Santos ${ }^{1}$, Olívia R. Pereira ${ }^{2}$, Luís Pedro ${ }^{3}$ and Maria João Sousa ${ }^{2, *}$ \\ 1 School of Agriculture, Polytechnic Institute of Bragança, Campus of Santa Apolónia, Apartado 1172, \\ 5301-855 Bragança, Portugal; a35307@alunos.ipb.pt \\ 2 Centro de Investigação de Montanha (CIMO), Instituto Politécnico de Bragança, Campus de Santa \\ Apolónia, 5300-253 Bragança, Portugal; oliviapereira@ipb.pt \\ 3 Centro de Estudos do Ambiente e do Mar (CESAM), Faculdade de Ciências da Universidade de Lisboa, \\ Centro de Biotecnologia Vegetal (CBV), C2, Piso 1, Campo Grande, 1749-016 Lisboa, Portugal; \\ lmpedro@ciencias.ulisboa.pt \\ * Correspondence: joaos@ipb.pt; Tel.: +351-273-303-200 \\ + Presented at the 1st International e-Conference on Antioxidants in Health and Disease, 01-15 December \\ 2020; Available online: https://cahd2020.sciforum.net/.
}

Published: 30 November 2020

\begin{abstract}
The Hops (Humulus lupulus L.) are known worldwide as an essential flavor in the beer industry. Its compounds have been showing health benefits in terms ofphytochemical, pharmacological and biological profiles, due to their antimicrobial, antioxidant, anti-inflammatory and anticancer activities. This study intends todevelop a gel formulation incorporating hydroalcoholic extracts of different varieties of hops such as Cascade, Polaris and Spontaneous, from the cones and thevegetative plant parts, in different percentages. The essential oil of Thymus zygis was used as a natural conservative and the analysis of the composition was made byGC and GC-MS. Later evaluation of the physical-chemical parameters, stability and capacity to inhibit microbial growth was made. The $\mathrm{pH}$ measurement results of theformulations tend to be adjusted to the natural $\mathrm{pH}$ skin. The relative density obtained values of 1 and 0.857 . It was found that there were no changes in the phase separation in the centrifugation, vibration, stability and accelerated test. However, changes were observed in the UV-Visible spectra, in texture, consistency and viscosity, and in the color test. In the light test there was phase separation in the samples, which may be related to the manufacturing technique of the formulations.However, this formulations anti aging with phenols from Hop can be development in order to obtain formulations with relevant properties for the consumers and the cosmetic industry.
\end{abstract}

Keywords: Humulus lupulus; cosmetics

\section{Introduction}

The Hops (Humulus lupulus L.) are known worldwide as an essential flavor in the beer industry, however in recent years, its compounds have been showing health benefits in terms of phytochemical, pharmacological and biological profiles, due to their antimicrobial, antioxidant, antiinflammatory and anticancer activities [1]. 
The 1st International Electronic Conference on Antioxidants in Health and Disease, 1-15 December 2020

In commercial and scientific terms in recent years there has been a substantial increase in the interest and development of cosmetic formulations and methodologies for maintaining a youthful appearance, reducing the signs of aging. Since very early in the history of mankind, extracts of plants and herbs have been used in this perspective since they are rich in bioactive compounds such as terpenes, alkaloids and phenols [2]. In addition to the importance of bioactive compounds present in the cosmetic formulation, it is also essential to use efficient preservatives, and there are aromatic and medicinal plants with antimicrobial activity already well described, as is the case with Thymus zygis. T. zygis is widespread throughout the world, and his flowers and leaves are used, which have antimicrobial properties for gram positive and gram negative bacteria as well as antioxidant capacity [3]. The main constituent of the essential oil of thyme is thymol (with greater activity), [4], can provide health benefits, related to the control of the reactive species production and oxidative stress and also in the prevention of aging and cell damage [5]. It was with this aim of being antimicrobial that the essential oil of thyme was used as a preservative in the cosmetic formulation.

This study intends to develop a gel formulation incorporating different percentages of hydroalcoholic extracts obtained from the cones and the vegetative parts of spontaneous hop and from Cascade and Polaris varieties. And to analyze the physical-chemical and microbiological stability of the formulation developed with these extracts.

\section{Experiments}

2.1.

Plant material: The samples of the Hops plant of the Polaris and Cascade variety were collected in the Quinta do Polão experimental field of the Escola Superior de Agrária of the Polytechnic Institute of Bragança. The Spontaneous Hops sample was collected in Bragança away from cultivated reas of commercial varieties. The sample of the plant Thymus zygis L. (Thyme) subsp. zygis, collected in Rebordãos, Bragança, Portugal. After being identified botanically, the samples were frozen at -20 ${ }^{\circ} \mathrm{C}$ until use in the extraction process.

\subsection{Development of Cosmetic Formulation}

The cosmetic formulation was developed based on the following recipe: Methylcellulose (3.7\%); 96\% Alcohol (18.9\%); Distilled water(78\%); Glycerin (0.13\%) Extract of Humulus lupulus (variable); and thymus zygis zygis(variable). The amount of hop extract depends on the concentration under study in each sample $(1.25 \% ; 2.5 \%$ and $5 \%)$, and in the case of Thymus the tested formulation samples have $0.013 \%$.

\subsection{Essential Oils Extraction}

The essential oil of aerial parts of Thymus zygis, used as a natural preservative of the cosmetic formulations, was obtained by hydrodestilation from the female cones and leaves using a LikensNickerson system, and analyzed by GC and GC-MS [6].

\subsection{Phenolic Compounds Extraction}

Solid-liquid extraction with an $80 \%$ etanolic solution was used to prepare the extracts from cones and vegetative parts of spontaneous hop and from Cascade and Polaris varieties. The phenolic compounds heve been determined used the Folin-Ciocalteu method [3].

\subsection{Stability Tests of the Cosmetic Formulation}

Physical-chemical parameters and stability were determined used differents methodologies:

- $\quad$ Analisies of the variations in $\mathrm{pH}$

- Determination of density 
The 1st International Electronic Conference on Antioxidants in Health and Disease, 1-15 December 2020

- $\quad$ Assay in the centrigfuge

- Assay in vortex

- Spectrophotometer determination

- Texture

- Light

- Tests of temperature

pH test: $3 \mathrm{~g}$ of the gel formulation was used and dissolved in distilled water for $\mathrm{pH}$ measurement with the help of a digital $\mathrm{pH}$ meter ( $\mathrm{pH} 7310 \mathrm{wtw}$ xylem brand inolab)

on day zero the $\mathrm{pH}$ of the gel was measured without the hydroalcoholic extracts and then with the spontaneous hop hydroalcoholic extracts and the Cascade and Polaris varieties, and also the control (methylcellulose gel, without extracts). The $\mathrm{pH}$ was measured at three days, one week and fifteen days after production.

Density: The relative density was determined by the formula:

$$
\text { Dr }=\mathrm{m} / \mathrm{v}
$$

Relative density (Dr), mass in grams (m) and volume in milliliters (v).

It was used as a way to determine the relative density, the water displacement method.

Centrifuge assay: To see the stability of the formulation, in which there may be a tendency to phase separation, we centrifuged at $25^{\circ} \mathrm{C}, 3000 \mathrm{RPM}$, for $30 \mathrm{~min}$ in an equipment (hettich mikro 24$48 \mathrm{r}$ zentrifugen) with one gram of each sample in eppendorfs of $1.5 \mathrm{~mL}$.

Assay in vortex (Mechanical vibration test): This test aims to replicate the transport conditions, indicating whether the vibration movements of the same can modify the sample. The procedure used was one gram of each sample in $1.5 \mathrm{~mL}$ eppendorfs submitted to 2000 RPM in vibration on a vortex shaker (Velp scientifica zx3 advance vortex mixer) for $10 \mathrm{~s}$.

Spectrophotometric test: The samples were diluted in distilled water and placed in quartz cuvettes and subsequently subjected to a spectrophotometric analysis in a spectrophotometer (vwr uv-1600pc spectrophotometer), with the spectrum being traced, in the UV-Visible region, between 210 and $600 \mathrm{~nm}$.

Texture: This test aims to demonstrate the cohesiveness, consistency, firmness and viscosity index of the samples that were made on a texture analyzer (Stable Micro Systems TA-XT Plus) and equipped with a probe coupled with a $35 \mathrm{~mm}$ compression disk that prints a force on a $50 \mathrm{~mm}$ diameter cylindrical container. The equipment operated at a height of $100 \mathrm{~mm}$, the gel column was $50 \mathrm{~mm}$ and occupied about $50 \%$ of the total volume of the container. After starting its movement, the compression disc exerts a force on the sample surface for a short period of time and descends to a depth of $20 \mathrm{~mm}$, at a constant speed, using a return speed of $20 \mathrm{~mm} \mathrm{~min}^{-1}$ until it returns the sample surface.

Light: In this test we placed fifteen grams of each type in sterile transparent plastic Petri dishes in which they were subjected to extreme lighting conditions, $16 \mathrm{~h}$ on and $8 \mathrm{~h}$ off, using a dayligth lamp, for 15 days.

Testo of temperature (Temperature and Humidity test) one gram each, and placed in $1.5 \mathrm{~mL}$ eppendorfs with three repetitions each, in total 57 eppendorfs and were stored in an incubator (memmert) at a temperature of $25{ }^{\circ} \mathrm{C} \pm 2{ }^{\circ} \mathrm{C}$ at $60 \% \pm 5 \%$ relative humidity (HR) for two weeks and at a temperature of $40 \pm 2{ }^{\circ} \mathrm{C}$ at $75 \% \pm 5 \% \mathrm{RH}$ during the same time. Organoleptic characteristics (color, smell, phase separation, texture and consistency) and the $\mathrm{pH}$ value every eight days were analyzed 
The 1st International Electronic Conference on Antioxidants in Health and Disease, 1-15 December 2020

\section{Results}

\subsection{Chemical Composition}

\subsubsection{Phenolic Composition of Hops Extracts}

In general, cone hydroethanolic extracts are richest in phenolic compounds than extracts obtained from vegetative parts. In fact, higher amounts of phenolic compounds have been determined in cone extracts of Polaris and Cascade varieties $(22.7 \pm 2.4$ and $10.4 \pm 2.1 \mathrm{mg} / \mathrm{GAE} \mathrm{g}$ of plant, respectively) comparing to the obtained for extracts of vegetative parts of the same plants (Table 1).

Table 1. Yield of extraction (\%) and total phenolic compounds (mg GAE/g plant) of a spontaneous and two variaties of hop.

\begin{tabular}{ccc}
\hline Sample & $\begin{array}{c}\text { Yeld } \\
\mathbf{( \% )}\end{array}$ & $\begin{array}{c}\text { Total Phenolic Compounds } \\
\text { (mg GAE/g Plant) }\end{array}$ \\
\hline Spontaneous C & 13.11 & ND \\
Spontaneous VP & 21.1 & $3.1 \pm 0.5^{2}$ \\
Cascade C & 30.3 & $10.4 \pm 2.1$ \\
Cascade VP & 9.3 & $2.1 \pm 0.7$ \\
Polaris C & 25.8 & $22.7 \pm 2.4$ \\
Polaris VP & 11.8 & $1.1 \pm 0.2$ \\
\hline${ }^{2}$ Mean values \pm SD; C-Cones; VP-Vegetative Parts; ND-Not determined.
\end{tabular}

\subsubsection{Thymus Zygis Essential oil Composition}

The yield of the essential oil of the aerial parts of Thymus zygis zygis, based on the dry mass of the plant, was as follows $1.14 \%$ (Table 2), in wich carvacrol, terpinen-4-ol, p-cymene and transsabinene hydrate were the main compounds $(43.60 \%, 25.80 \%, 24.10 \% 15.8 \%$, respectivelly).

Table 2. Essential oil composition of aerial parts of Thymus zygis zygis.

\begin{tabular}{cc}
\hline Components & Amount \\
\hline cis-linalool oxide & 0.60 \\
cis-sabinene hydrate & 0.10 \\
camphor & 3.20 \\
borneol & 1.2 \\
terpinen-4-ol & 25.8 \\
$\alpha$-terpineol & 11.8 \\
thymol & 0.30 \\
carvacrol & 43.60 \\
$\alpha$-thujene & 1.60 \\
$\alpha$-pinene & 0.80 \\
camphene & 1.00 \\
3-octanol & 1.00 \\
$\beta$-myrcene & 1.00 \\
$\alpha$-terpinene & 1.40 \\
p-cymene & 24.10 \\
limonene & 0.20 \\
trans- $\beta$-ocimen & 1.10 \\
trans-sabinene hydrate & 15.80 \\
Yeld $(\%, v / w)$ & 1.14 \\
\hline
\end{tabular}

Mean values. 
The 1st International Electronic Conference on Antioxidants in Health and Disease, 1-15 December 2020

3.1.3. Stability Tests

pH test: The control formulation (Gel) showed a slightly acidic pH value. However, after fifteen days, all formulations with varieties showed a $\mathrm{pH}$ trend between 7.5 to 6.5 .

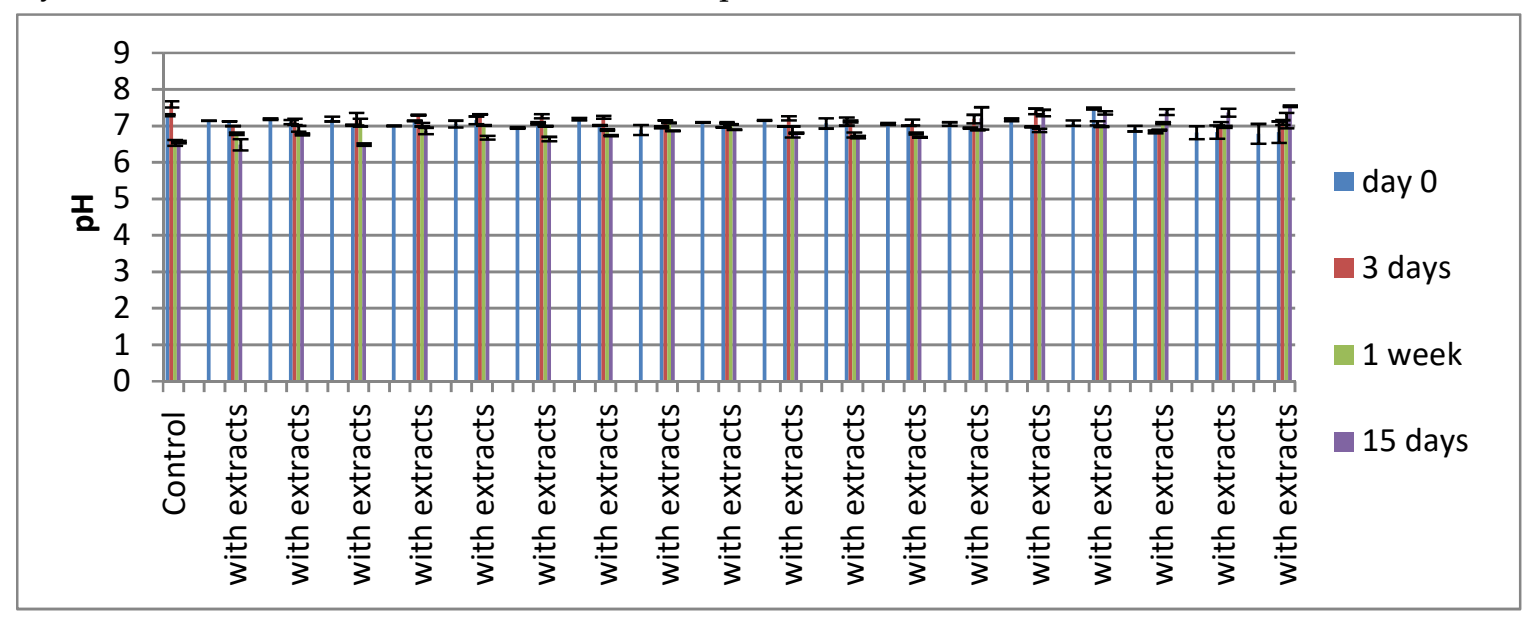

Figure 1. In the graph is possible to see that que $\mathrm{pH}$ is very stable and slightly acidic.

Density test: In the measurement of relative density values of 1 and 0.857 were obtained, (Table 2 ), so it can be concluded that the relative density of the formulation with different concentrations with the spontaneous hops and the Polaris variety both of flower, was found the obtained value is 0.857, which is lower compared to the gel and the other formulations.

Table 3. Measurement of relative density.

\begin{tabular}{|c|c|c|c|c|}
\hline & \multicolumn{4}{|c|}{ Relative Density } \\
\hline & $\begin{array}{l}\text { Weight } \\
\text { (g) }\end{array}$ & $\begin{array}{l}\text { Initial Volume } \\
(\mathrm{ml})\end{array}$ & $\begin{array}{l}\text { Final Volume } \\
(\mathrm{ml})\end{array}$ & $\begin{array}{l}\text { Relative } \\
\text { Density }\end{array}$ \\
\hline Gel (control) & 3 & 15 & 18 & 1 \\
\hline \multicolumn{5}{|l|}{$\begin{array}{l}\text { Spontaneous } \\
\text { vegetative }\end{array}$} \\
\hline $1.25 \%$ & 3 & 15 & 18 & 1 \\
\hline $2.5 \%$ & 3 & 15 & 18 & 1 \\
\hline $5 \%$ & 3 & 15 & 18 & 1 \\
\hline \multicolumn{5}{|c|}{ Spontaneous flower } \\
\hline $1.25 \%$ & 3 & 15 & 18.5 & 0.857 \\
\hline $2.5 \%$ & 3 & 15 & 18.5 & 0.857 \\
\hline $5 \%$ & 3 & 15 & 18.5 & 0.857 \\
\hline \multicolumn{5}{|c|}{ Cascade vegetative } \\
\hline $1.25 \%$ & 3 & 15 & 18 & 1 \\
\hline $2.5 \%$ & 3 & 15 & 18 & 1 \\
\hline $5 \%$ & 3 & 15 & 18 & 1 \\
\hline \multicolumn{5}{|l|}{ Cascade flower } \\
\hline $1.25 \%$ & 3 & 15 & 18 & 1 \\
\hline $2.5 \%$ & 3 & 15 & 18 & 1 \\
\hline $5 \%$ & 3 & 15 & 18 & 1 \\
\hline \multicolumn{5}{|l|}{ Polaris vegetative } \\
\hline $1.25 \%$ & 3 & 15 & 18 & 1 \\
\hline $2.5 \%$ & 3 & 15 & 18 & 1 \\
\hline $5 \%$ & 3 & 15 & 18 & 1 \\
\hline Polaris flower & & & & \\
\hline
\end{tabular}


The 1st International Electronic Conference on Antioxidants in Health and Disease, 1-15 December 2020

\begin{tabular}{ccccc}
$1.25 \%$ & 3 & 15 & 18.5 & 0.857 \\
$2.5 \%$ & 3 & 15 & 18.5 & 0.857 \\
$5 \%$ & 3 & 15 & 18.5 & 0.875 \\
\hline
\end{tabular}

- $\quad$ Centrifuge assay: There was no phase separation.

- Assay in vortex (Mechanical vibration test): There was no phase separation.

- Spectrophotometric test: This test aims to see the behavior of the gel if it is different when adding extracts of the varieties in different concentrations, verifying this in the occurrence of changes in the intensity of the bands (hyperchromic effects (increase in absorption intensity) or hypochromic (decrease in absorption intensity))) or variations in the wavelength at which maximum absorption occurs (bathochromic effects (change in absorption for longer wavelengths, due to solvent replacement or effect) or hypsochromic (change in absorption for shorter wavelengths, due to substitution or effect of the solvent)) indicate the occurrence of a change in its color or intensity, which may be an indication of instability of the formulation.

In Figure 2 (graph), it can be seen that in the nineteen spectra the absorption bands are in the same position, verifying the existence of a maximum absorption peak, at $300 \mathrm{~nm}$ and $350 \mathrm{~nm}$. These peaks are due to the compounds in the formulation used, which possibly have the phenols functional groups, which have typical bands at these wavelengths. In the formulations there is a hyperchromic effect compared to the gel. This change is possibly due to the proportions of some compounds, which translates into a change in the intensity of the bands. It can also be seen that there is a hypsochromic effect in which it is respective due to the low wavelengths that are located closer to the blue.

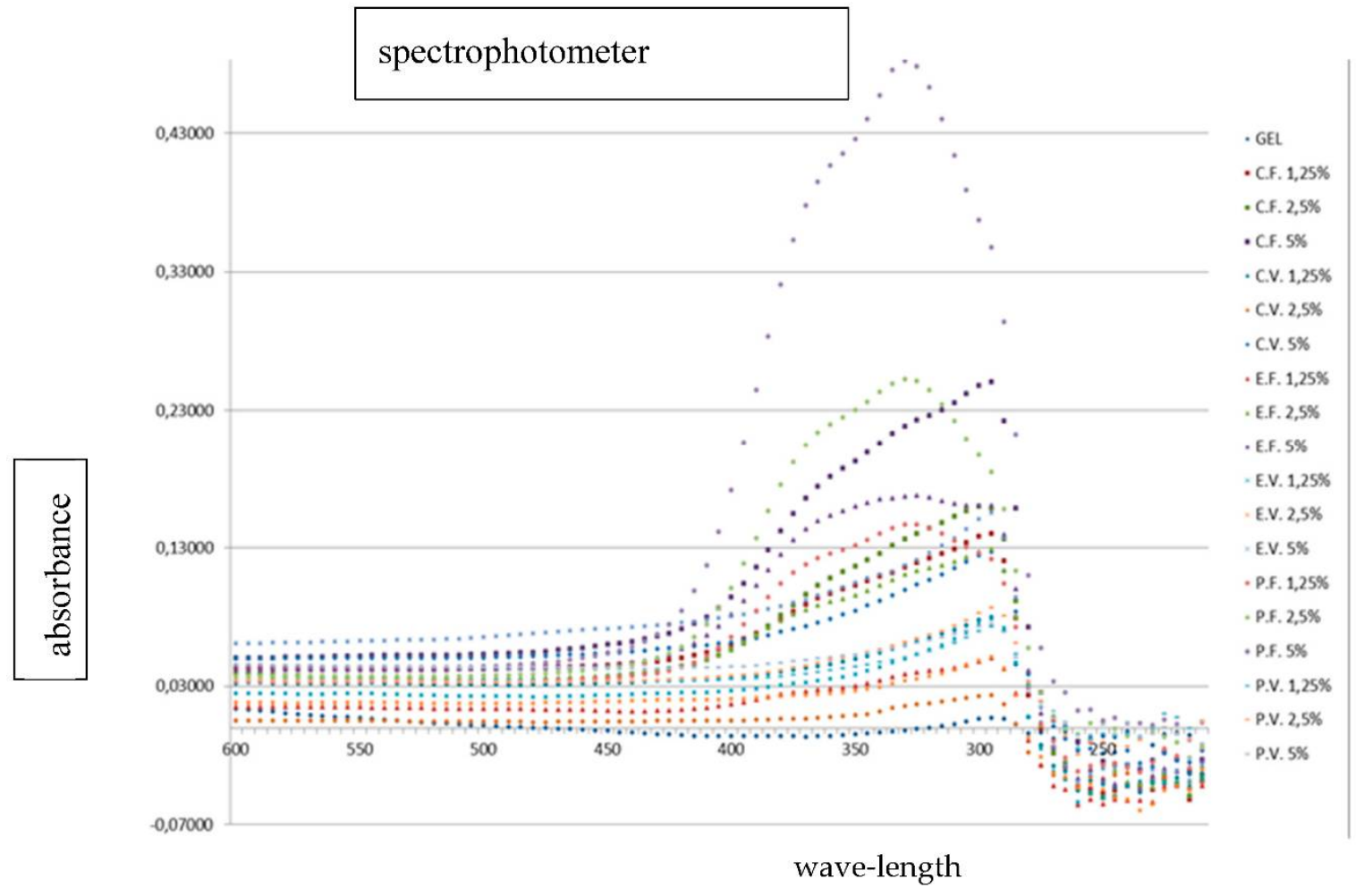

Figure 2. Grafic pf the UV-VIS spectrum of samples in different concentrations; C.F. - Cascade flower; C.V.-Vegetative cascade; E.F.-Spontaneous flower; E.V.-Spontaneous vegetative; P.F.-Polaris flower; P.V.-Vegetative polaris.

Temperature test: After this period, it was verified whether the organoleptic characteristics of each sample (color, odor, phase separation, texture and consistency), in which they were compared with the samples in their natural/initial state and at the end And there was no change, in the organoleptic aspect or in the $\mathrm{pH}$ value. 
The 1st International Electronic Conference on Antioxidants in Health and Disease, 1-15 December 2020

Texture: The texture of the various gel samples was evaluated using a texturometer, through the performance of reverse extrusion tests (back extrusion), with recording of the parameters firmness, consistency, viscosity index, and cohesiveness. The graph below (Figure 3)show the results provided by the texturometer software for the samples, making it possible to identify the parameters recorded by the equipment.

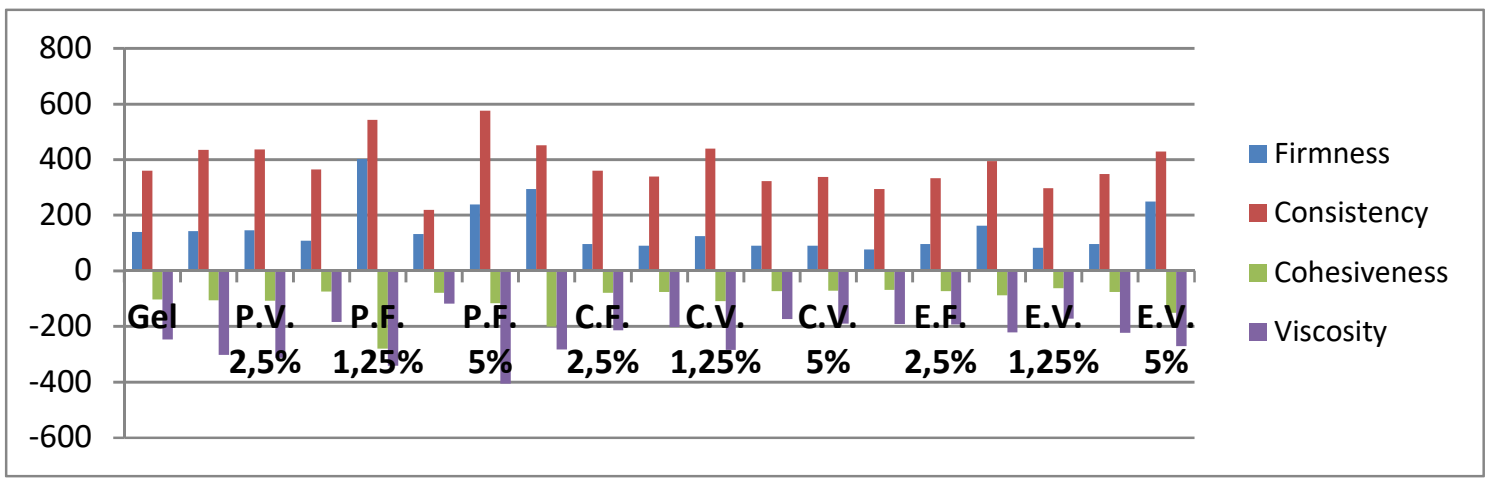

Figure 3. In the graph it is possible to observe the values of the parameters measured by the equipment, verifying that only the formulation with the extracts of the Polaris Variety deviates a little more from the remaining values. Featuring higher consistency values, and less viscosity.

\section{Discussion}

The results presented are still preliminary in the sense that several parameters have yet to be determined, including the effectiveness of the buoyant compounds on the skin and also the toxicity of the extracts, however the hop extracts are not referred to as having skin toxicity, so interesting perspectives are opened for the use of these extracts and formulations.

\section{Conclusions}

In conclusion the gels are stable in time and can be used as a main part of cosmetic formulation by further research. Concerning its bioactivity Humulus lupulus showed an optimistic stability physico-chemical and appropriate organoleptic characteristics, maintaining over time (more than 4 months) its microbiological stability which allows, together with microbiological data (data not shown) already developed in this study to ensure that stability. The the continuity of this work involves developing more toxicity tests and other stability tests and testing on cell lines and sensory analysis panels, in order to analyze the possibility of this formulation, and others with hops extracts, may have beneficial effects and effective in skin health.

Author Contributions: M.J.S., L.P. and O.R.P. conceived and designed the experiments; B.d.S. performed the experiments and analyzed the data. M.J.S. contributed reagents and materials; M.J.S., L.P. and O.R.P. reviewed and edited. All authors have read and agreed to the published version of the manuscript.

Acknowledgments: The authors thank the Foundation for Science and Technology (FCT, Portugal) and the ERDF under the PT2020 Program for their financial support to CIMO (UIDB/00690/2020).

Conflicts of Interest: The authors declare no conflict of interest.

\section{References}

1. Astray, G.; Gullón, P.; Gullón, B.; Munekaba, P.E.S.; Lorenzo, J.M. Humulus lupulus L. as a Natural Source of Functional Biomolecules. Appl. Sci. 2020, 10, 5074, doi:10.3390/app10155074.

2. Binic, I.; Lazarevic, V.; Ljubenovic, M.; Mojsa, J.; Sokolovic, D. Skins Ageing: Natural Weapons and Stratagies. Evid. Based Complementary Altern. Med. 2013, 2013, doi:10.1155/2013/827248. 
The 1st International Electronic Conference on Antioxidants in Health and Disease, 1-15 December 2020

3. Rota, M.; Herrera, A.; Martínez, R.; Sotomayor, J.; Jordán, M. Antimicrobial activity and chemical composition of Thymus vulgaris, Thymus zygis and Thymus hyemalis essential oils. Food Control 2008, 19, 681687.

4. Lee, S.; Umano, K.; Shibamoto, T.; Lee, K. Identification of volatile components in basil (Ocimum basilicum L.) and thyme leaves (Thymus vulgaris L.) and their antioxidant properties. Food Chem. 2005, 91, 131-137.

5. Martins, N.; Barros, L.; Ferreira, I.C. Antioxidant activity of decoction, infusion and methanolic extract of cultivated thyme (Thymus vulgaris L.); EJI, Polytechnic Institute of Bragança: Bragança ,Portugal, 2013.

6. Figueiredo, M.V.B.; Martinez, C.R.; Burity, H.A.; Chanway, C.P. Plant growth-promoting rhizobacteria for improving nodulation and nitrogen fixation in the common bean (Phaseolus vulgaris L.). World J. Microbiol. Biotechnol. 2008, 24, 1187-1193, doi:10.1007/s11274-007-9591-4.

7. Singleton, V.L.; Rossi, J.A. Colorimetry of total phenolics with phosphomolybdic-phosphotungstic acid reagents. Am. J. Enol. Vitic. 1965, 16, 144-158.

8. Afonso, A.F.; Pereira, O.R.; Válega, M.; Silva, A.M.S.; Cardoso, S.M. Metabolites and Biological Activities of Thymus zygis, Thymus pulegioides, and Thymus fragrantissimus Grown under Organic Cultivation. Molecules 2018, 23, 1514, doi:10.3390/molecules23071514.

Publisher's Note: MDPI stays neutral with regard to jurisdictional claims in published maps and institutional affiliations.

(C) 2020 by the authors: Licensee MDPI, Basel, Switzerland. This article is an open access article distributed under the terms and conditions of the Creative Commons by Attribution (CC-BY) license (http://creativecommons.org/licenses/by/4.0/). 